\title{
Espaço público na cidade contemporânea: inventário dos espaços públicos de lazer na área continental do município de Florianópolis 1
}

\section{Public space in the modern city: inventory of leisure facilities in the continental area of the municipality of Florianópolis}

\author{
Costa, Guilherme 1; Agostinho, Maria da Graça2; Duarte, Ana Alice Miranda ${ }^{3}$ \\ 1 Universidade do Sul de Santa Cataria, Rua Manoel Leôncio de Souza Brito, 1781, \\ Vargem Pequena - Florianópolis/SC, Brasil, e-mail: guilherme.csta@gmail.com \\ 2 Universidade do Sul de Santa Cataria, e-mail:mg.agostinho3@gmail.com \\ 3 Universidade do Sul de Santa Cataria, e e-mail: anaalice.mduarte@gmail.com
}

\section{RESUMO}

O presente trabalho faz parte de uma ampla pesquisa que está sendo desenvolvida, no qual objetiva-se elaborar uma avaliação dos espaços públicos de lazer na porção continental do município de Florianópolis, Santa Catarina. Trata-se da elaboração de um inventário constituído de um amplo levantamento e mapeamento das praças públicas, produzindo um banco de dados com o cadastramento e avaliação de cada área pública de lazer. Foi adotado um sistema geográfico de informação para a criação de um banco de dados a fim de organizar as informações bem como mapear os espaços públicos de lazer. Propõe-se avaliar a qualidade das condições materiais da morfologia das praças bem como, das condições de sua apropriação pela população.

Palavras-chave: espaços públicos de lazer, sistema geográficos de informação, avaliação de espaços livres.

\begin{abstract}
The present work is part of an extensive research that is being developed, in which the objective is to elaborate an evaluation of the public leisure spaces in the continental portion of the municipality of Florianópolis, Santa Catarina. It is the elaboration of an inventory consisting of a wide survey and mapping of public squares, producing a database with the registration and evaluation of each public leisure area. A geographic information system was adopted for the creation of a database in order to organize the information as well as to map the public leisure spaces. It is proposed to evaluate the quality of the material conditions of the morphology of the squares as well as the conditions of their appropriation by the population.
\end{abstract}

Keywords: Leisure public spaces, geographic information system, free space evaluation.

\footnotetext{
${ }^{1}$ COSTA, Guilherme; AGOSTINHO, Maria da Graça; DUARTE, Ana Alice Miranda. Espaço público na cidade contemporânea: inventário dos espaços públicos de lazer na área continental do município de Florianópolis. In: II SIMPÓSIO NACIONAL DE GESTÃO E ENGENHARIA URBANA: SINGEURB, 2019, São Paulo. Anais... Porto Alegre: ANTAC, 2019.
} 


\section{INTRODUÇÃO}

O sistema de espaços públicos constitui-se em uma estrutura fundamental na organização das cidades. As ruas, avenidas, praças, parques, entre outros é o suporte material sobre o qual se apoia a grande duração que assegura a permanência nas cidades e configuram o contexto arquitetônico da vida pública urbana.

Segundo Gehl (2015), o arranjo espacial das cidades pode convidar ou repelir pessoas para uma vida pública. Há muitos exemplos de como a renovação de um espaço, ou mesmo a mudança no mobiliário urbano, a arborização, entre outros detalhes, pode convidar as pessoas a desenvolver um padrão de uso do espaço público totalmente novo.

A importância do espaço público se dá na medida que as pessoas se conectam com o lugar. Segundo Serpa (2007), é principalmente a história do indivíduo que determina sua relação com os espaços que compõem o seu cotidiano. O lugar transforma e vira história pessoal e coletiva.

Um espaço é uma realidade, ao mesmo tempo, objetiva e subjetiva. Um bairro não é socialmente produzido somente porque seu substrato material (as ruas, as construções) representa a natureza transformada pelo trabalho humano, mas porque cada bairro é, ao mesmo tempo e em última análise, um espaço dotado de significado, um espaço vivido e percebido (SOUZA, 2013).

Segundo Lamas (2004, p.102), a praça é um elemento morfológico importante nas cidades ocidentais. Para o autor,

A definição de praça na cidade tradicional implica, como na rua, a estreita relação do vazio (espaços de permanência) com os edifícios, os seus planos marginais e as fachadas. Estas definem os limites da praça e caracterizam-na, organizando o cenário urbano. A praça reúne a ênfase do desenho urbano como espaço coletivo de significação importante. Este é um dos seus atributos principais e que a distingue dos outros vazios da estrutura das cidades.

O presente trabalho faz parte de uma ampla pesquisa que está sendo desenvolvida, no qual objetiva-se elaborar uma avaliação dos espaços públicos de lazer na área continental do município de Florianópolis, Santa Catarina. Trata-se da elaboração de um inventário constituído de um amplo levantamento e mapeamento das praças públicas, produzindo um banco de dados com o cadastramento e avaliação de cada área pública de lazer. Propõese avaliar a qualidade das condições materiais da morfologia das praças bem como, das condições de sua apropriação pela população.

Sistemas geográficos de informação podem ser uma ferramenta importante no mapeamento e cadastramento de áreas nas cidades. Eles servem como suporte no estudo para tornar as análises dos corpos técnicos mais velozes (SOUZA, 2013). Através dessa ferramenta digital de levantamento, mapeamento e avaliação de espaços públicos foi possível identificar, localizar e analisar as praças na área continental de Florianópolis. Inicialmente constatou-se o abandono dos espaços públicos, uma das possíveis causas para a baixa apropriação desses lugares pela população local.

Segundo dados do IBGE 2018, o município de Florianópolis possui uma população estimada para o ano de 2018 de 492.977 pessoas e possui $674,844 \mathrm{~km}^{2}$ de área territorial, desta área, aproximadamente, $98 \%$ pertence à llha de Santa Catarina. A porção continental (Figura 1) possui cerca de $12,1 \mathrm{~km}^{2}$ de área territorial e uma população de cerca de 100 mil pessoas, segundo a ACIF - Associação Comercial e Industrial de Florianópolis. 
Figura 1 - Mapa distrital de Florianópolis

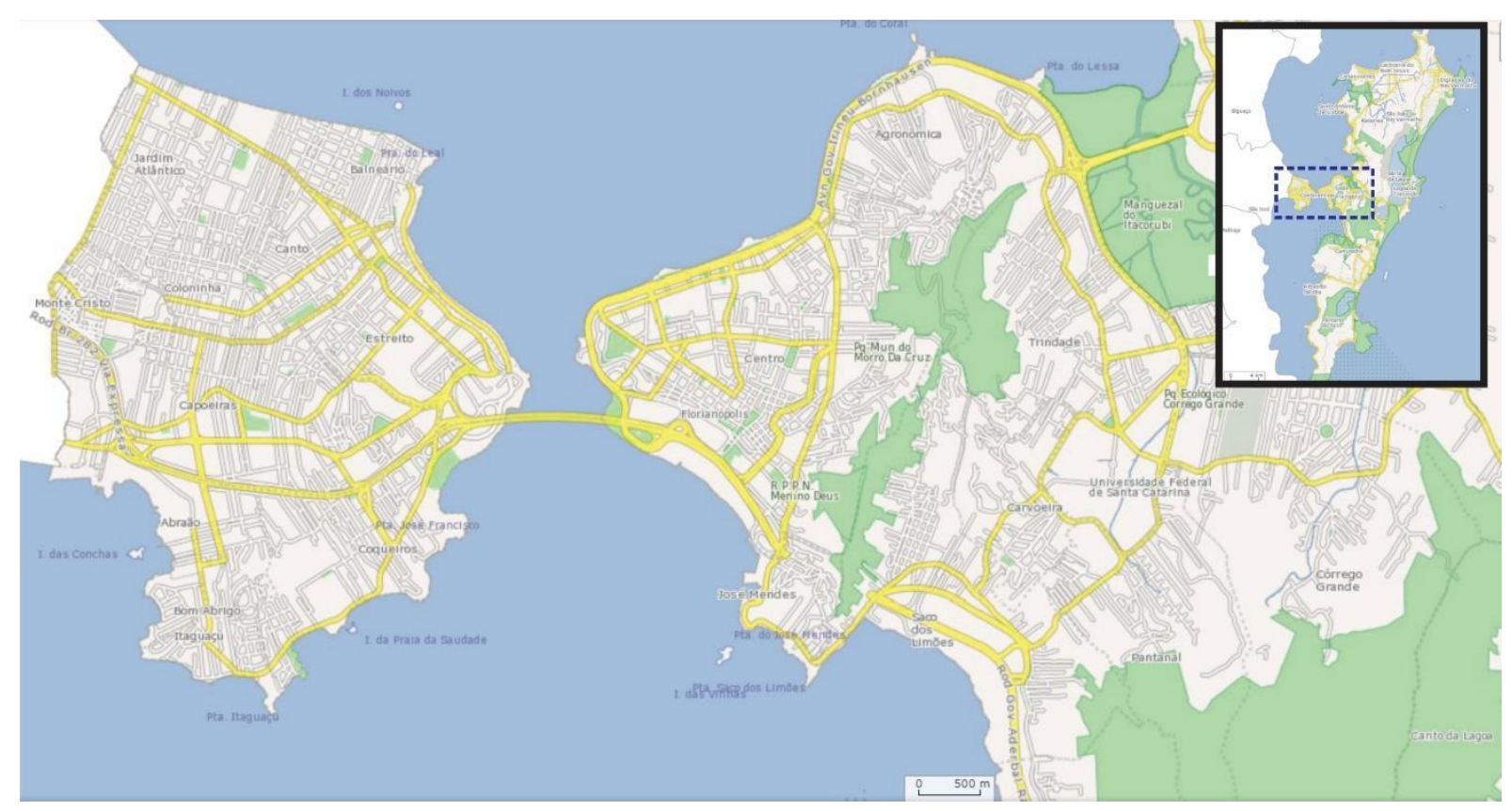

Fonte: Geoprocessamento Corporativo - Prefeitura Municipal de Florianópolis (2019)

\section{CADASTRAMENTO DE ÁREAS}

O cadastramento de áreas ou espaços específicos dentro dos bairros da cidade possui caráter importante no estudo de políticas públicas e auxílio no planejamento urbano das cidades. O geoprocessamento pode ser de enorme utilidade como coadjuvante do esforço de otimização no emprego de cadastros, pois a informação dos bancos de dados permite agilidade na recuperação de dados, atrelado a experiência profissional na análise dos dados armazenados.

O geoprocessamento e os sistemas Geográficos de Informação (SGIs) não são, propriamente, instrumentos de planejamento, no sentido que um tributo ou um zoneamento o são. Antes, o geoprocessamento e os SGls constituem um suporte tecnológico para o trabalho em planejamento urbano, com o qual estudos, avaliações de impactos, monitoramento da evolução de fenômenos, simulações e a representação cartográfica de unidades de manejo podem ganhar em agilidade e precisão (SOUZA, 2013, p. 310).

A ausência de dados de cadastramento pode dificultar atividades corriqueiras no planejamento das cidades, atividades simples como abastecimento de dados no sistema SGIs podem acelerar estudos para aplicação de um urbanismo paramétrico, tomada de decisões de benefício a políticas públicas e métodos e estudos de avaliação de sistema de espaços livres na cidade.

\section{ELABORAÇÃO DE CADASTRAMENTO DE ESPAÇOS LIVRES PÚBLICOS NA PORÇÃO CONTINENTAL DE FLORIANÓPOLIS.}

A partir da necessidade de conhecer a realidade referente aos espaços livres da cidade de Florianópolis e, especialmente, identificação dos espaços públicos de lazer no recorte da área continental do município, foi realizada, inicialmente, um amplo levantamento de dados a partir de revisão bibliográfico; consulta ao geoprocessamento corporativo da Prefeitura Municipal de Florianópolis, site que identificou alguns espaços públicos de lazer; bem como, foi consultado, no site da Câmara de Vereadores, as leis municipais, especialmente as leis que denominam e criam praças e parques na cidade. Identificou-se na porção continental do município de Florianópolis 37 espaços públicos de lazer, entre praças, parques e bosques. Esse 
levantamento inicial permitiu não só identificar a quantidade de praças, mas também a sua área, localização e lei de criação, conforme demonstrado na Tabela 1.

Tabela 1 - Tabela dos espaços públicos de lazer levantados, área continental de Florianópolis

\begin{tabular}{|c|c|c|c|}
\hline NOME & TAMANHO $\left(\mathrm{m}^{2}\right)$ & BAIRRO & LEI \\
\hline Praça Manoel Felix Cardoso & - & Abraão & - \\
\hline Praça dos Navegantes & $2.900,00$ & Balneário Estreito & $5122 / 97$ \\
\hline Praça Senador Renato Ramos da Silva & $12.528,00$ & Balneário Estreito & $1650 / 79$ \\
\hline Praça Anízio da Silveira Machado & $2.700,00$ & Canto & $4716 / 95$ \\
\hline Praça Antonieta de Barros e Felipe Neves & - & Canto & - \\
\hline Praça do Canto & - & Canto & - \\
\hline Praça Humaitá & - & Canto & - \\
\hline Praça Eugênio Melo ou Eugênio Cordeiro Dutra & $1.235,00$ & Capoeiras & - \\
\hline Praça Nagib Jobor & $3.289,00$ & Capoeiras & - \\
\hline Praça Osvaldo de Oliveira & 315,00 & Capoeiras & $2047 / 84$ \\
\hline Praça Patricio Caldeira de Andrade & - & Capoeiras & - \\
\hline Praça Paulo Schlemper & - & Capoeiras & $942 / 70$ \\
\hline Praça A & - & Coqueiros & - \\
\hline Praça Manoel David Chaves ou Praia do Meio & $11.494,33$ & Coqueiros & $4949 / 96$ \\
\hline Praça B & - & Coqueiros & - \\
\hline Praça Duque de Caxias & $1.000,00$ & Estreito & - \\
\hline Praça Alziro Zarur & $2.380,00$ & Estreito & $2070 / 84$ \\
\hline Praça João DeMaria Cavalazzi & 875,00 & Estreito & $1821 / 81$ \\
\hline Praça Nossa Senhora de Fátima & $8.866,00$ & Estreito & - \\
\hline Parque Infantil Itaguaçu & - & Itaguaçu & - \\
\hline Praça Calistrato Salles & 225,00 & Itaguaçu & $2843 / 88$ \\
\hline Praça Conselho Comunitário Sapé & - & Jardim Atlântico & - \\
\hline Praça Arlindo Phillipe & - & Jardim Atlântico & $6562 / 04$ \\
\hline Praça Eduardo Dias & - & Jardim Atlântico & - \\
\hline Praça João Batista Vieira & $3.184,00$ & Jardim Atlântico & - \\
\hline Praça Pe. Marcelo Champaunat & - & Jardim Atlântico & - \\
\hline Praça Marcílio Dias & $1.850,00$ & Jardim Atlântico & $329 / 58$ \\
\hline Praça Monte Cristo & - & Monte Cristo & - \\
\hline Praça Guilherme Fortekamp & 900,00 & Balneário Estreito & - \\
\hline $\begin{array}{l}\text { Praça Aquiles Paulo de Souza/ Praça Jardim } \\
\text { Atlântico }\end{array}$ & 450,00 & Jardim Atlântico & $3032 / 88$ \\
\hline Praça Marcelino Vieira Filho & 30,00 & Canto & $3540 / 91$ \\
\hline Praça Roberto Oliveira & $2.994,10$ & Estreito & $1787 / 81$ \\
\hline Bosque Vereador Pedro Medeiros & $10.107,66$ & Estreito & $3409 / 90$ \\
\hline Praça Cientista Dr. Albert Sabin & - & Coqueiros & $4044 / 93$ \\
\hline Praça Coronel Romariz & - & Balneário Estreito & $6034 / 02$ \\
\hline Praça Pública Professor Ângelo Ribeiro & - & Estreito & $6685 / 05$ \\
\hline Parque Aventura Maria Inês Tournier Rodrigues & $34.049,93$ & Abraão/ Coqueiros & $6806 / 05$ \\
\hline
\end{tabular}

Fonte: Os autores.

Num segundo momento, foi elaborado um cadastro padrão para aplicação no momento da visita in loco e posterior análise de cada praça identificada e mapeada. Tomando como ponto de partida o trabalho sobre representação percentual dos equipamentos existentes em espaços públicos na cidade de Arapiraca-AL, (GOMES et al., 2012), chegou-se ao seguinte modelo, ver Figura 2: 
Figura 2 - Cadastro padrão: espaços públicos de lazer na área Continental de Florianópolis.

FORMULÁRIO DE ANÁLISE DE ESPAÇOS PÚBLICOS DE LAZER NA ÁREA

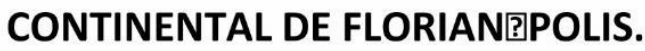

Data do levantamento: l

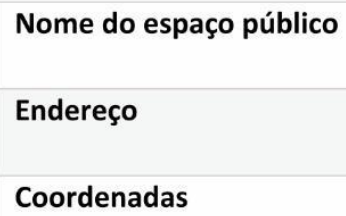

\section{Habitação percepção visual}

$\square$

$\square$ Alto Padrão (Acima 600k)

$\square$ Médio Padrão (250k a 600k)

Médio/ Baixo Padrão (MCMV)

Baixo Padrão (Até 3 Salários)

\section{Aspecto visual e} manutenção do espaço

$\square$ ótimo

$\square$ Bom

$\square$ Regular

$\square$ Ruim

$\square$ Péssimo
Topografia do terreno

Íngreme

Plano / Íngreme

Muito íngreme

\section{Forma geométrica}

Retangular

Circular

$\square$ Triangular

$\square$ Orgânica

$\square$ Outra 
EQUIPAMENTOS EXISTENTES

\begin{tabular}{|c|c|c|c|c|c|}
\hline EQUIPAMENTO & SIM & NÃO & Quant. & ESTADO & OBSERVAÇÕES \\
\hline \multicolumn{6}{|l|}{ Iluminação } \\
\hline \multicolumn{6}{|l|}{ Bancos } \\
\hline \multicolumn{6}{|l|}{ Equipamento Infantil } \\
\hline \multicolumn{6}{|l|}{ Calçadas } \\
\hline \multicolumn{6}{|l|}{ Pista corrida/ caminhada } \\
\hline \multicolumn{6}{|l|}{ Lixeiras } \\
\hline \multicolumn{6}{|l|}{ Sanitários } \\
\hline \multicolumn{6}{|l|}{ Bebedouros } \\
\hline \multicolumn{6}{|l|}{ Estrutura esportiva } \\
\hline \multicolumn{6}{|l|}{ Telefone público } \\
\hline \multicolumn{6}{|l|}{ Banca de Revistas } \\
\hline \multicolumn{6}{|l|}{ Quiosque } \\
\hline \multicolumn{6}{|l|}{ Pessoa com deficiência } \\
\hline \multicolumn{6}{|l|}{ Ponto de Táxi } \\
\hline \multicolumn{6}{|l|}{ Ponto de ônibus } \\
\hline \multicolumn{6}{|l|}{ Estacionamento } \\
\hline \multicolumn{6}{|l|}{ Espaço Espetáculos } \\
\hline \multicolumn{6}{|l|}{ Espaço infantil cercado } \\
\hline \multicolumn{6}{|l|}{ Estruturas Idosos } \\
\hline \multicolumn{6}{|l|}{ Placa Inauguração } \\
\hline \multicolumn{6}{|l|}{ Obras de Arte Pública } \\
\hline \multicolumn{6}{|l|}{ Espelho d'água } \\
\hline \multicolumn{6}{|l|}{ Espaço Pet } \\
\hline \multicolumn{6}{|l|}{ Espaço para eventos } \\
\hline \multicolumn{6}{|l|}{ Pavimentação } \\
\hline \multicolumn{6}{|l|}{ Piso podo tátil } \\
\hline \multicolumn{6}{|l|}{ Segurança Pública } \\
\hline \multicolumn{6}{|l|}{ Câmeras de segurança } \\
\hline \multicolumn{6}{|l|}{ Bicicletário } \\
\hline Quadras Esportivas & & & & & \\
\hline
\end{tabular}

VEGETAÇÃO E PAISAGISMO

\begin{tabular}{|c|c|c|c|c|}
\hline TIPO & SIM & NÃO & Quant. & ESPÉCIE \\
\hline \multicolumn{5}{|c|}{ Árvore de grande porte } \\
\hline \multicolumn{5}{|c|}{ Árvore de médio porte } \\
\hline \multicolumn{5}{|c|}{ Árvore de pequeno porte } \\
\hline \multicolumn{5}{|c|}{ Árvores frutíferas } \\
\hline \multicolumn{5}{|l|}{ Espécies Exóticas } \\
\hline \multicolumn{5}{|l|}{ Estratos Arbustivos } \\
\hline \multicolumn{5}{|l|}{ Gramado } \\
\hline \multicolumn{2}{|l|}{$\begin{array}{l}\text { Jardins (floreiras)/ } \\
\text { herbáceas }\end{array}$} & & & \\
\hline
\end{tabular}

Fonte: Os autores. 
Avaliação de situação das áreas públicas de lazer, tamanho, qualidade da infraestrutura, investimentos públicos, bem como caracterização dos espaços ou mesmo a relação entre a quantidade de pessoas e áreas verdes podem ser analisadas com base nos dados apresentados para a área continental, podendo esse ser um modelo facilmente aplicado em outras localidades. Afim de verificar e tipificar a morfologia dos espaços, bem como sua localização, o resultado das primeiras visitas in loco foi testar a pertinência do cadastro. Tendo em vista a necessidade de encontrar o tamanho de algumas áreas, utilizou-se o programa de banco de dados e análise de georreferenciamento QGIS 2.18.18. Configura-se um mapa (Figura 3) com a seguinte formatação inicial, onde as demarcações em verde são as praças analisadas e a tabela com os dados do cadastro padrão (Figura 3) sistematizado em banco de dados.

Figura 3 - Modelo do resultado da sistematização das informações das praças levantadas.

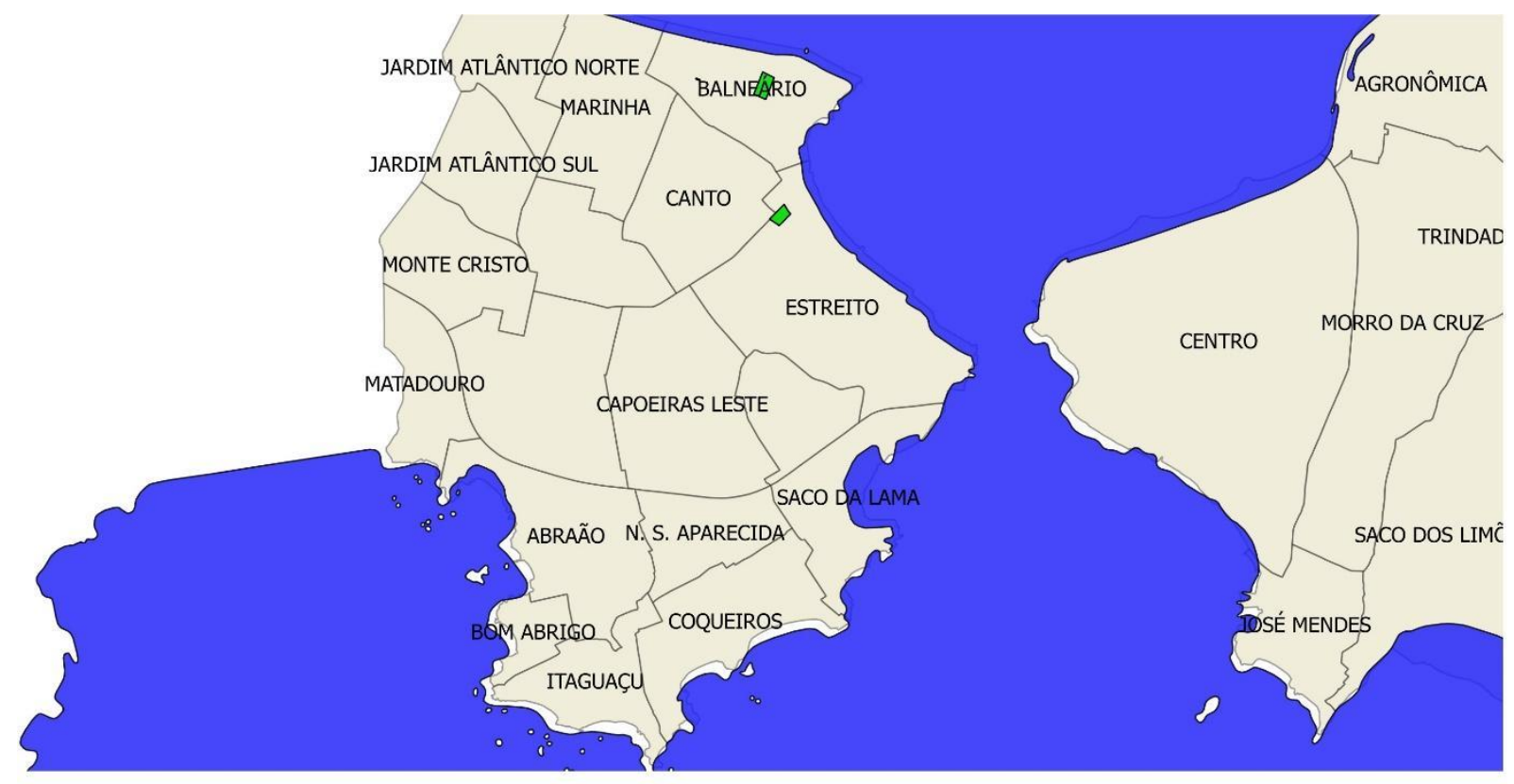

\begin{tabular}{|l|l|l|l|l|l|l|l|l|l|}
\hline Praça & Endereço & Bairro & Dimensão & Latitude & Longitude & Entorno & Topografia & Geometria & Estado Vis \\
\hline Praça Nossa Senhora de Fátima & Rua Fúlvio Aducci & Estreito & 8564.06 & $27^{\circ} 35^{\prime \prime} 11^{\prime \prime S}$ & $48^{\circ} 34^{\prime} 46^{\prime \prime} \mathrm{W}$ & Médio Padrão & Plano/ Íngrime & Retangular & Bom \\
\hline Praça Guilherme Fortekamp & Vila da Caixa Econômica - Rua Sérgio Gil & Balneário Estreito & 163.56 & $27^{\circ} 34^{\prime} 45^{\prime \prime} \mathrm{S}$ & $48^{\circ} 35^{\prime} 07,3^{\prime \prime} \mathrm{W}$ & Médio Padrão & Plano & Retangular & Ruim \\
\hline Praça Senador Renato Ramos da Silva & Rua Sérgio Gil & Balneário Estreito & 12555.31 & $27^{\circ} 34^{\prime} 45,1^{\prime \prime} \mathrm{S}$ & $48^{\circ} 34^{\prime} 515^{5^{\prime \prime} \mathrm{W}}$ & Médio Padrão & Plano & Retangular & Bom \\
\hline
\end{tabular}

Fonte: Os autores.

\section{CONSIDERAÇÕES FINAIS}

Foi constatado a ausência de pesquisas e dados relativos a qualidade dos espaços públicos de lazer no município de Florianópolis. Desta forma, verificou-se a importância da pesquisa, a fim de nortear as decisões de gestão e planejamento urbano, no que tange a relação de utilização e conservação dos espaços públicos, bem como a qualidade de vida e apropriação das áreas públicas de lazer pela população.

Não foram identificados sistemas de cadastramento de banco de dados atualizados referentes aos espaços públicos nos órgãos públicos. Alguns dos dados encontrados eram incompletos, tais como, tamanhos de praças e a lei de criação de alguns espaços públicos.

Na verificação in loco, aplicando o cadastro (Figura 3), foi identificado que algumas praças possuem nomes diferentes dos que constam nos registros. Em uma análise mais completa quanto ao local, algumas praças não estão configuradas como tal, constituem-se em espaços vazios, sem manutenção, investimento ou qualquer tipo de apropriação pelas pessoas no seu entorno imediato. 
O cadastramento e atualização desse banco de dados se faz necessário para que estudos sejam realizados e para que seja dado a devida atenção a esses lugares de vida pública, com segurança e qualidade.

\section{REFERÊNCIAS}

CABRAL, Thalyne Nadja Dittert. ESPAÇO PÚBLICO E URBANIDADE: UM ESTUDO SOBRE A APROPRIAÇÃO DE PRAÇAS NO MUNICÍPIO DE FLORIANÓPOLIS. 2015. 263 f. Dissertação (Mestrado) - Curso de Arquitetura e Urbanismo, Programa de Pós-Graduação em Urbanismo, História e Arquitetura da Cidade - Pgau-cidade, Universidade Federal de Santa Catarina, Florianópolis, 2015.

FLORIPAMANHÃ, Associação. A cidade de Florianópolis possui 95 Praças Públicas. 2011. Disponível em: <http://floripamanha.org/201 1/08/a-cidade-de-florianopolis-possui-95-pracaspublicas/>. Acesso em: 07 abr. 2019.

GEHL, Jan. Cidades Para Pessoas. 3. ed. São Paulo: Editora Perspectiva S.a., 2015. 262 p.

GOMES, Marcos Antônio Silvestre et al. CARACTERIZAÇÃO E ANÁLISE dOS ESPAÇOS PÚBLICOS DA CIDADE DE ARAPIRACA-AL-BRASIL. Ateliê Geográfico: Revista Eletrônica, Goiânia - Go, v. 4, n. 6, p.137-157, dez. 2012. Disponível em: Acesso em: 25 maio 2018.

IBGE - Instituto Brasileiro de Geografia e Estatística. Cidades e Estados: Florianópolis. 2018. Disponível em: <https://www.ibge.gov.br/cidades-e-estados/sc/florianopolis.html? >. Acesso em: 07 abr. 2019.

LAMAS, José Manuel Ressano Garcia. Morfologia Urbana e Desenho da Cidade. 3. ed. Lisboa: Orgalimpressores-porto, 2004. 590 p.

SERPA, Angelo. O espaço público na cidade contemporânea. 2. ed. São Paulo: Editora Contexto, 2007. 205 p.

SOUZA, Marcelo Lopes de. Mudar a Cidade: Uma Introdução Crítica ao Planejamento e à Gestão Urbanos. 9. ed. Rio de Janeiro: Editora Bertrand Brasil Ltda, 2013. 558 p.

VAZ, Murad Jorge MusSi. A PRODUÇÃO DO ESPAÇO PÚBLICO EM FLORIANÓPOLIS: AS PRAÇAS E A VIDA URBANA. 2016. 520 f. Tese (Doutorado) - Curso de Geografia, Programa de Pós Graduação em Geografia, Universidade Federal de Santa Catarina, Florianópolis, 2015. 